\title{
Novel Biosensor Detection of Tuberculosis Based on Photonic Band Gap Materials
}

\author{
Arafa H Aly, (1), D. Mohamed ${ }^{a}$, Z. A. Zaky , Z. S. Matar , N. S. Abd El-Gawaad ${ }^{c}$, A. S. Shalabyd, \\ Fatima Tayeboun ${ }^{e}$ M. Mohaseb ${ }^{a, b}$ \\ ${ }^{a}$ Beni-Suef University, Faculty of Sciences, Physics Department, TH-PPM Group, Beni Suef, Egypt \\ ${ }^{b}$ Umm-Al-Qura University, Faculty of Applied Science, Department of Physics, Mecca, Saudi Arabia \\ ${ }^{c}$ King Khalid University, Faculty of Science, Abha, Saudi Arabia \\ ${ }^{d}$ Beni-Suef University, Faculty of Sciences, Physics Department, Beni Suef, Egypt \\ ${ }^{e}$ Djillali LIABES University, Sidi-Bel-Abbes 22000, Algeria
}

Received: October 19, 2020; Revised: February 18, 2021; Accepted: March 21, 2021

\begin{abstract}
Tuberculosis is one of the most widespread infectious and deadliest diseases in the world. The death percentage is larger than that in the case of the current Coronavirus. Bio-photonic sensors represent a promising option for developing reliable, simple, and affordable tools for the effective detection of tuberculosis. In this paper, a novel design of an optical biosensor will be used as a tuberculosis detector based on a resonance cavity with high sensitivity in one-dimensional photonic crystals demonstrated theoretically. The results show that the increase of the defect layer thickness shifts the defect mode to a longer wavelength region. Besides, it is shifted to a shorter wavelength region via the increase of the incidence angle. The change in thickness of the defect layer and incident angle of light cause an optimization for our suggested structure and the sensitivity reaches $1390 \mathrm{~nm} / \mathrm{RIU}$. Our structure is very simple for industrial design.
\end{abstract}

Keywords: Tuberculosis, refractive index, photonic crystal, detector, sensitivity, biosensor.

\section{Introduction}

Photonic crystals (PCs) recently become one of the amazing favorite methods used in integrated optical circuits ${ }^{1,2}$, and many authors employing PCs in many valuable applications ${ }^{3-10}$. Photonic crystals consist of a multilayer of dielectric materials with low and high refractive indices that control the propagation of light. Photonic crystals have a very interesting region called the Photonic bandgap region (PBG) which is the frequency ranges that are not allowed to propagate in the structure ${ }^{11,12}$. By introducing the defects inside the photonic structure, scientists can control and adjust the PBG. Accordingly, many optical devices are produced such as optical filters ${ }^{13,14}$, optical switches ${ }^{15,16}$, photonic crystal fibers ${ }^{17-19}$.

Tuberculosis (TB) is one of the deadly serious and most widespread infectious disease ${ }^{20}$. According to the World Health Organization (WHO), in 2017 there are around 1.6 million TB deaths, including 300,000 people living with $\mathrm{HIV}^{21}$. In 2019, data were informed by 202 countries and territories that account for more than $99 \%$ of the world's population and roughly calculate the number of TB cases ${ }^{21}$. An accurate diagnosis very early on active pulmonary TB is crucial for the control of the disease and treatment of infected patients. It is very important to develop new techniques for detecting TB diagnosis because a large proportion of all TB cases $(98 \%)$ in developing countries occur without access to biomedical analysis laboratories. Optical biosensors are considered as analytical detectors that transduce the blood reactions/interactions into an optical signal ${ }^{22}$. The biophotonic sensors have many advantages that distinguished them from

*e-mail: arafa.hussien@science.bsu.edu.eg conventional diagnostic techniques, such as single-step detection, ease of use, and most of the biosensor enabling better and rapid control over the measurement ${ }^{22}$. There are many types of biosensors such as electrical, piezoelectric, electrochemical, nanomechanical, magnetic, acoustic, and optical ${ }^{23-26}$. The optical transducer mechanism is the system that modulates the interaction with optical radiation in a detectable way ${ }^{22}$. As a particular photonic structure that can be used for sensing is a photonic crystal (PC), we develop a highly sensitive biosensor structure based on 1D-PC with a defect layer. The proposed design can be fabricated by many different ways as reported in literatures ${ }^{27-31}$.

We calculate the transmittance for s-polarized light by utilizing the transfer matrix method (TMM) and studying the effect of some parameters to achieve the highest performance of the sensor configuration.

\section{Theoretical Model}

In this part, we consider a 1D-PC structure consists of Air/

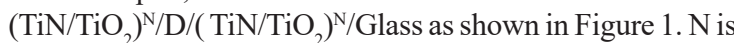
the number of periods. This structure consists of two dielectric media of thickness $d_{1}$ and $d_{2}$ with refractive indices $n_{1}$ and $n_{2}$. The structure is defected by a cavity between two identical periodic structures filled with various concentrations of TB blood samples. The thickness of this cavity is $\mathrm{d}_{3}$ with a refractive index $n_{3}$. Our structure is kept between the air and a substrate layer (glass). These materials $\left(\mathrm{TiN} / \mathrm{TiO}_{2}\right)$ are matching with the experimental work by Wei et al. ${ }^{32}$. We can assume in this stage $d_{3}=209 \mathrm{~nm}$. We have different concentrations of blood samples for TB patients with different refractive indices from 


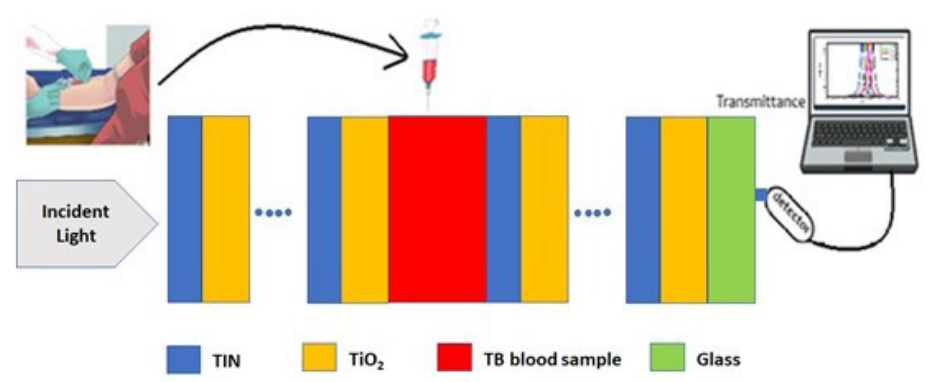

Figure 1. Schematic representation of the bio-photonic sensor structure.

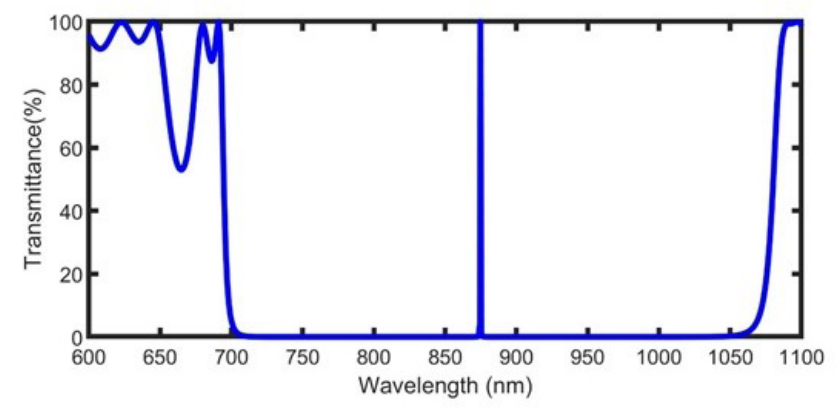

Figure 2. Transmittance spectra of defected 1DPC with a normal blood sample (TBN) at N=7, incident angle $\theta=0^{0}$, and the thickness of defect layer $d_{3}=209 \mathrm{~nm}$.

experimental work by Reddy et al. ${ }^{33}$. We change the thickness of the defect layer and incident angle for s-polarized light to optimize the structure performance. By utilizing TMM, we can obtain the interaction between the incident light and the structure by the following equations ${ }^{5,34-36}$ :

$M=\left(\begin{array}{ll}m_{11} & m_{12} \\ m_{21} & m_{22}\end{array}\right)=D_{o}^{-1}\left(D_{1} P_{1} D_{1}^{-1} D_{2} P_{2} D_{2}^{-1}\right)^{N} D_{s}$,

$M_{l}=\left[\begin{array}{cc}\cos \left(k_{l z} d_{l}\right) & j q_{l}^{-1} \sin \left(k_{l z} d_{l}\right) \\ j q_{l} \sin \left(k_{l z} d_{l)}\right. & \cos \left(k_{l z} d_{l}\right)\end{array}\right]$,

where, $M_{l}$ is the matrix for individual layer $l(l=\mathrm{A}, \mathrm{B}, \mathrm{C})$. then, the transmittance can be obtained based on the matrix elements to be:-

$T=\left|2 p \div\left(\left(M_{11}+M_{12} p\right) p+M_{21}+M_{22} p\right)\right|^{2}$,

$p=n \cos \left(\theta_{o}\right)$.

Thus, the reflectance is given by:-

$R=1-T$

The transmittance $(\mathrm{T})$ and the reflectance $(\mathrm{R})$ are obtained according to the following relations ${ }^{12,37}$.

$R=|r|^{2}=\left|\frac{M_{21}^{2}}{M_{11}}\right|$,

$T=|t|^{2}=\left|{\frac{1}{M_{11}}}^{2}\right|$.
The ratio of the change in the location of defect mode $(\Delta \lambda)$ concerning the change in the refractive index of the defect layer $(\Delta \mathrm{n})$ is called the sensitivity:

$s=\frac{\Delta \lambda}{\Delta n}$.

The refractive index and peak resonant position of the normal blood sample are used as references to calculate $\Delta \lambda$ and $\Delta \mathrm{n}$ for the different TB blood samples.

\section{Results and Discussion}

In Figure 2, we calculate the transmission spectrum of the structure. It illustrates the relation between transmittance spectra and wavelength range for defective 1D-PC at a thickness of the defect layer equals $d_{3}$. The thickness of TiN $\left(d_{1}\right)$ is $100 \mathrm{~nm}$ with refractive index $\mathrm{n}_{1}=1.2887$ and the thickness of $\mathrm{TiO}_{2}\left(\mathrm{~d}_{2}\right)$ is $109 \mathrm{~nm}$ with refractive index $\mathrm{n}_{2}=2.6143$. Also, we utilize the normal blood sample as a defect layer at the number of periods $\mathrm{N}=7$ and normal incidence $\theta=0^{0}$. The PBG is noticed in Figure 2 shared between the visible and infrared region with left and right edges $\lambda_{L}=710 \mathrm{~nm}$, and $\lambda_{R}=1038 \mathrm{~nm}$, respectively. The bandwidth of the PBG is equal to $328 \mathrm{~nm}$. The electromagnetic waves cannot propagate through structure at this PBG range except at only one wavelength which is called defect resonant mode. We can observe this defect peak in the PBG region with a high transmission rate reaches $99.99 \%$.

Now, we add the infected TB samples (TB1, TB2, TB3, and TB4) as a defect layer within the present structure, and then we can obtain different results as shown in Figure 3. The significance of the defect layer in Figure 3 illustrates a 
blue-shift in the position of the defect mode with changing the defect layer from TB1 to TB4 due to the decrease of the refractive indices of these samples, according to Table 1. The most infected sample (TB1) the least refractive index. This behavior is matching the condition of standing wave $\mathrm{e}^{39}$ :

$\Delta=m \lambda=n_{e f f} G$,

where $\Delta$ is the optical path difference, $\mathrm{m}$ is an integer, $\lambda$ is the wavelength of the incident light, $\mathrm{n}_{\text {eff }}$ is the effective refractive index of the defective 1D-PC, and G is the geometric path difference. The sensitivity is approximately $242.5 \mathrm{~nm} / \mathrm{RIU}$ at incident angle $\theta=0^{\circ}$, the number of periods $\mathrm{N}=7$ periods, and defected layer thickness is $\mathrm{d}_{3}=209 \mathrm{~nm}$.

\subsection{The selectivity of the sensor}

Both Table 1 and Figure 3 clear the high selectivity of the proposed sensor for different diseases because each disease has a different value of the refractive index. As Polyacrylamide has the highest value of the refractive index, the defect peak that represents it appeared at the highest wavelength at $900.7 \mathrm{~nm}$. Then the defect peaks of the cancer cells appeared from $888.7 \mathrm{~nm}$ (for MCF-7) to $886.1 \mathrm{~nm}$ (for Jurkat). Also, the defect peak continues to blue-shifts for hemoglobin to $883.7 \mathrm{~nm}$ as a result of the decreasing value of the refractive index of it. Finally, the peaks of TB infected samples appeared at the lowest wavelength because it has the lowest value of refractive indices. So, the proposed sensor has high selectivity.

\subsection{The effect of the defect layer thickness}

In this part, we will study the effect of increasing the TB sample layer thickness. If we changed the defect layer thickness within the structure from $209 \mathrm{~nm}$ to $1000 \mathrm{~nm}$, we will notice that the number of defect peaks increases and shifted to a high wavelength region with the increase of the thickness of the defect layer as shown in Figure 4. This result agrees with previous works ${ }^{39-41}$. Therefore, we will use high thicknesses for the defect layer and compare them to get a sensor with high sensitivity. It is clear in Figure 4 that the resonant peak is shifted to a high wavelength region by increasing the defect layer thickness until it gets out from the PBG range and another peak enters the PBG.

It is clear from Figure 5 that sensitivity increases rapidly to be $\mathrm{S}=650 \mathrm{~nm} / \mathrm{RIU}$ by increasing the defect layer thickness from $\mathrm{d}_{3}=209 \mathrm{~nm}$ to $\mathrm{d}_{3}=6000 \mathrm{~nm}$. Also, a few increasing in the sensitivity to $S=687.5 \mathrm{~nm} / \mathrm{RIU}$ is noticed when the defect layer thickness increased from $d_{3}=6000 \mathrm{~nm}$ to $d_{3}=12000 \mathrm{~nm}$. This behavior is matching the experimental and theoretical work in reference $\mathrm{e}^{39,40,42}$. From the foregoing, increasing the geometric path difference of the defect layer leads to increasing sensitivity

Table 1. The refractive index of different blood constituents $\mathrm{s}^{33,38}$.

\begin{tabular}{cc}
\hline Name of blood constituent & Refractive index \\
\hline Polyacrylamide & 1.452 \\
\hline MCF-7 cancer & 1.401 \\
\hline MDA-MB-231 cancer & 1.399 \\
\hline PC12 cancer & 1.395 \\
\hline HeLa cancer & 1.392 \\
\hline Jurkat cancer & 1.390 \\
\hline Hemoglobin & 1.380 \\
\hline TBN & 1.351 \\
\hline TB1 & 1.348 \\
\hline TB2 & 1.347 \\
\hline TB3 & 1.345 \\
\hline TB4 & 1.343
\end{tabular}

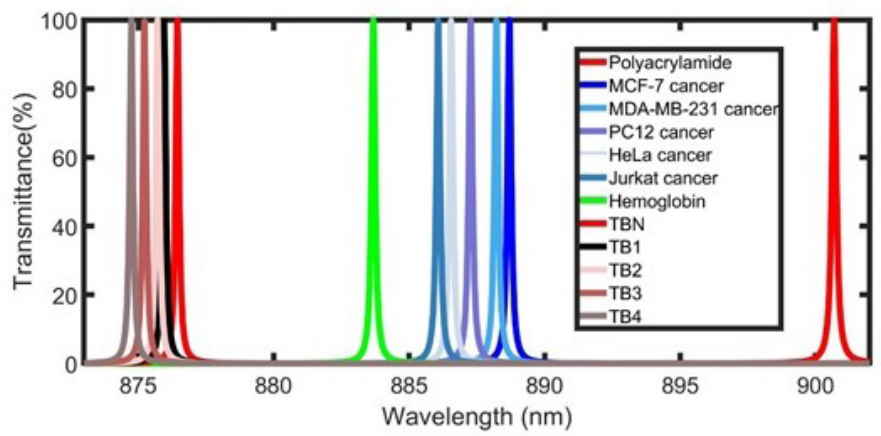

Figure 3. Transmittance spectra of defected $\mathrm{PC}$ with different blood components at $\mathrm{N}=7, \theta=0^{0}$ and $\mathrm{d}_{3}=209 \mathrm{~nm}$.

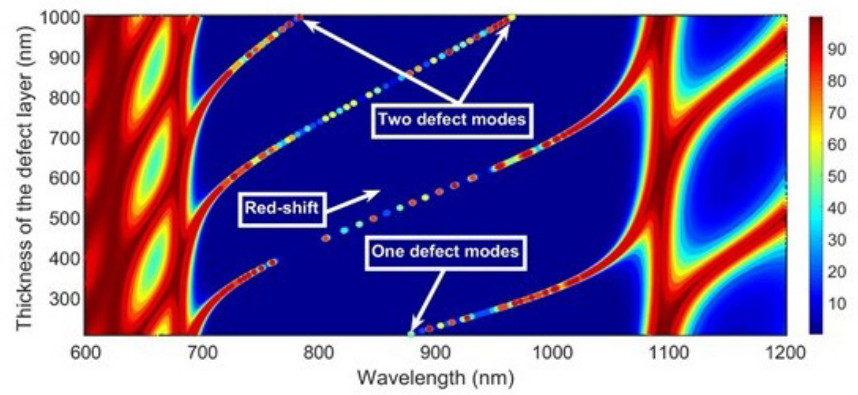

Figure 4. Transmittance spectra of defected 1D-PC by increasing the thickness of the defect layer for TBN at $\mathrm{N}=7$ and incident angle $\theta=0^{\circ}$. 


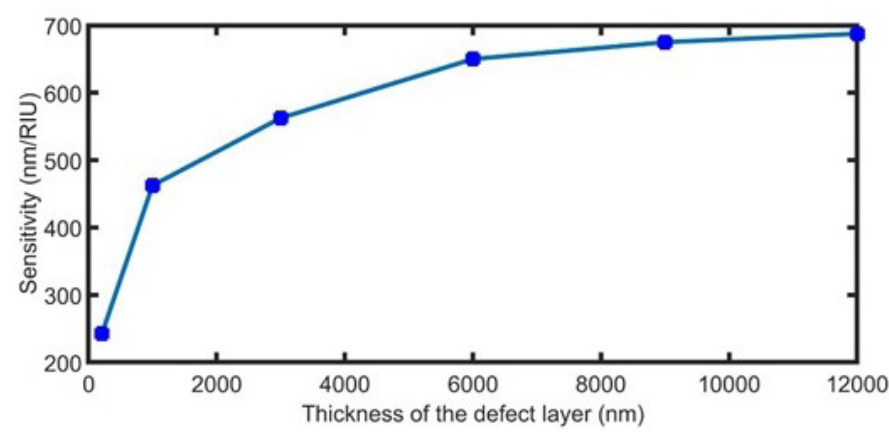

Figure 5. The effect of increasing the defect layer thickness on sensitivity at normal incidence.

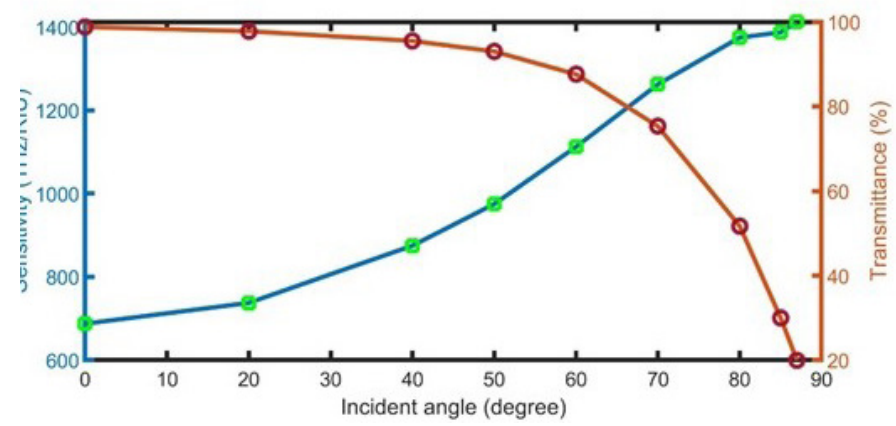

Figure 6. The effect of changing the incident angle on the sensitivity at $d_{3}=12000 \mathrm{~nm}$, and $\mathrm{N}=7$.

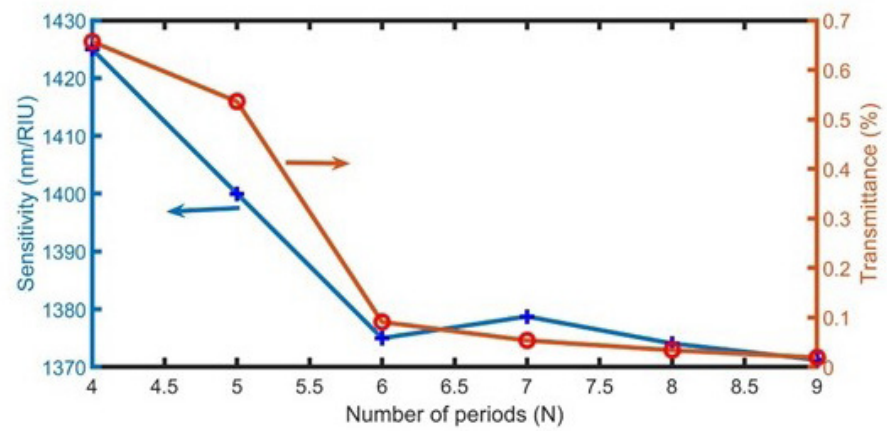

Figure 7. The effect of the number of periods in the performance of the sensor at $\theta=80^{\circ}$ and $\mathrm{d} 3=1200 \mathrm{~nm}$.

due to improving the interactions between light and blood TB samples ${ }^{42}$. Hence, strong electric field confinement is associated with defective 1D-PC and thus enhances the performance of the defective1D-PC as a biosensor. The maximum defect layer thickness which we considered in the proposed structure is $\mathrm{d}_{3}=12000 \mathrm{~nm}$ because another increase in the thickness causes the appearance of new peaks on the region of the electromagnetic wave spectra with a very small increase in the sensitivity.

\section{Effects of Incident Angle}

Here, the effect of changing the incident angle of the electromagnetic wave on the sensitivity of the proposed sensor will be studied. Figure 6 illustrates the behavior of the proposed sensor by changing the incident angle. At a certain defect layer thickness, $d_{3}=12000 \mathrm{~nm}$, the increasing of the incident angle shifts the position of defect mode to a lower wavelength region. The behavior in these results can be explained based on Bragg-snell law ${ }^{40}$. $m \lambda=2 d \sqrt{n_{e f f}^{2}-\sin ^{2}} \theta$,

where $\mathrm{m}$ is the constructive diffraction order, $\lambda$ is the wavelength of the maximum reflected intensity, $d$ is the period, $n_{\text {eff }}$ is the effective refractive index of the defective $1 \mathrm{D}-\mathrm{PC}$, and $\theta$ is the angle of incidence. By increasing the incident angle in Figure 6, the sensitivity of the proposed sensor rapidly increases from $\mathrm{S}=687.5 \mathrm{~nm} / \mathrm{RIU}$ at $\theta=0^{0}$ to be $\mathrm{S}=1375 \mathrm{~nm} / \mathrm{RIU}$ at $\theta=80^{\circ}$. On the other hand, the transmittance of the defect peak decreases from $99 \%$ to $51 \%$. For more increase in the incident angle, the transmittance decreases to $20 \%$ with a small increase in the sensitivity. So, the angle of $\theta=80^{\circ}$ will be the optimum angle.

\section{Effects of the Number of Periods}

As clear in Figure 7, by increasing the number of periods from 4 to 6 , the peaks begin to be sharp, and the 


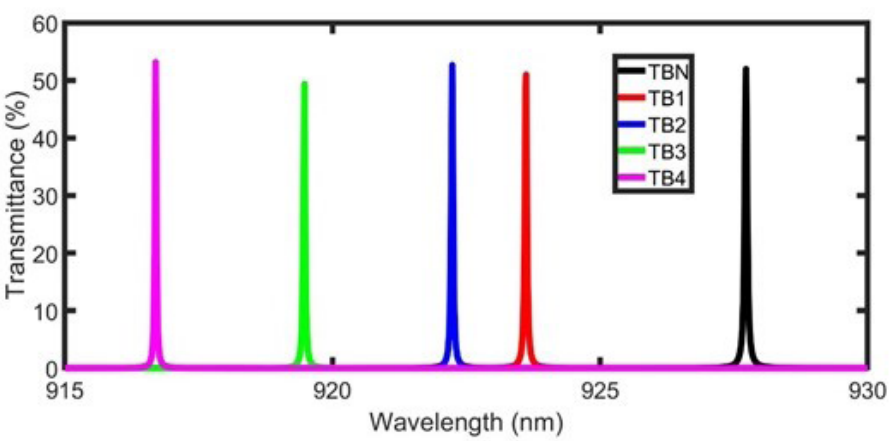

Figure 8. Transmittance spectra of defected PC with different blood samples of tuberculosis patients at $\mathrm{N}=7, \theta=80^{\circ}$ and $\mathrm{d}_{3}=12000 \mathrm{~nm}$.

Table 2. the performance parameters of the proposed sensor.

\begin{tabular}{|c|c|c|c|c|c|c|}
\hline \multirow{2}{*}{ TB sample } & $\mathrm{N}$ & $\lambda_{d}$ & $\mathrm{~S}$ & & FoM & LoD \\
\hline & (RIU) & $(\mathrm{nm})$ & (nm/RIU) & $\times 10^{4}$ & $\times 10^{4}(\mathrm{RIU})$ & $(\mathrm{RIU}) \times 10^{6}$ \\
\hline TBN & 1.351 & 927.7 & & 15762 & 0 & --- \\
\hline TB1 & 1.348 & 923.6 & 1370 & 20525 & 3.0 & 1.6 \\
\hline TB2 & 1.347 & 922.2 & 1380 & 22884 & 3.4 & 1.5 \\
\hline TB3 & 1.345 & 919.45 & 1380 & 26271 & 3.9 & 1.3 \\
\hline TB4 & 1.343 & 916.7 & 1390 & 28620 & 4.3 & 1.2 \\
\hline
\end{tabular}

Table 3. Comparing the sensitivity of the proposed sensor with other biosensing designs.

\begin{tabular}{lcc}
\hline \multicolumn{1}{c}{ Design } & S (nm/RIU) & Year, Ref. \\
\hline defected ring mirror 1D-PC for detecting mycobacterium tuberculosis bacteria. & 700 & $2019^{46}$, \\
\hline Graphene-based leaky-wave structure for blood plasma sensing & 431 & $2020^{47}$, \\
\hline 1D-PC for hemoglobin sensing & 167 & $2020^{48}$, \\
\hline 1D-PC waveguide & 51 & $2019^{49}$, \\
\hline Our work & 1390 & \\
\hline
\end{tabular}

bandwidth (FWHM) sharply decreases from $0.66 \mathrm{~nm}$ to $0.02 \mathrm{~nm}$. Besides, the sensitivity decreases. For $\mathrm{N}$ higher than 6 periods, both sensitivity and FWHM seem to be constant. The number of periods $\mathrm{N}=7$ will be optimum. The sensitivity reaches $1379 \mathrm{~nm} / \mathrm{RIU}$, and the FWHM is $0.02 \mathrm{~nm}$.

The defect modes should have a high-quality factor for high-performance biosensor design. By calculating the quality factor, it leads to accurate sensor measurements. The quality factor can be calculated by this equation:

$Q=\frac{\lambda_{d}}{F W H M}$,

where $\lambda_{d}$ is the resonant wavelength and FWHM is the full width at half maximum of the transmission peak of defect mode. The resonant peaks become broader for the high TB concentration sample. From our proposed structure the simulation results reveal that the suggested biosensor has a Q-factor of about $2 \times 10^{4}$ as shown in Table 2. It is known that a high Q-factor means a sharp resonant peak ${ }^{43}$. The ratio of sensitivity to the full width at half maximum of the resonant peaks is called the figure of merit (FoM):

$F o M=\frac{S}{F W H M}$.
As clear in Table 2, the FoM value of the sensor is about $3.5 \times 10^{4}$ RIU. Finally, the limit of detection for our suggested sensor is calculated from this equation ${ }^{44,45}$.

$L o D=\lambda /(20 \times S \times Q)$

As clear in Figure 8 and Table 2, LoD equals $1.5 \times 10^{-0}$ RIU. The value of LoD is very low that means the proposed sensor is good as it capable to resolve very small changes in the refractive index.

As clear in Table 3, the sensitivity of our proposed structure is higher than that previously reported for other structures. In 2020, Ramanujam et al.studied a theoretical sensitivity of a defected ring mirror 1D-PC for detecting mycobacterium tuberculosis bacteria. They achieved a sensitivity of $700 \mathrm{~nm} /$ RIU. The proposed structure achieved twice the value of sensitivity.

\section{Conclusion}

In the current work, we have simply constructed a theoretical strategy for a photonic detector via a 1D-PC consists of alternating layers to detect tuberculosis disease. The effect of different tuberculosis blood concentrations, changing the thickness of the defect layer, angle of the incident light, and the number of periods on the performance of the 
proposed design was studied. By changing the concentrations of the tuberculosis blood sample from normal to infected samples, the defect peak is shifted to a lower wavelength region. Increasing the defect layer thickness and the angle of incidence enhance the sensitivity of the proposed sensor and the sensitivity reached $1390 \mathrm{~nm} / \mathrm{RIU}$ and the LoD is about $1.5 \times 10^{-6}$ RIU.

\section{References}

1. McNab SJ, Moll N, Vlasov YA. Ultra-low loss photonic integrated circuit with membrane-type photonic crystal waveguides. Opt Express. 2003;11:2927-39.

2. Saghaei H, Zahedi A, Karimzadeh R, Parandin F. Line defects on As2Se3-Chalcogenide photonic crystals for the design of all-optical power splitters and digital logic gates. Superlattices Microstruct. 2017;110:133-8.

3. Joannopoulos JD, Villeneuve PR, Fan S. Photonic crystals: putting a new twist on light. Nature. 1997;386:143-9.

4. Aly AH, Mohamed D, Mohaseb M. Metamaterial control of hybrid multifunctional High-Tc superconducting photonic crystals for 1D Quasi-periodic structure potential applications. Mat Res. 2020;23(3):e20190695.

5. Shaban SM, Mehaney A, Aly AH. Determination of 1-propanol, ethanol, and methanol concentrations in water based on a onedimensional phoxonic crystal sensor. Appl Opt. 2020;59:3878-85.

6. Nouman WM, Abd ElGhany S, Sallam SM, Dawood AFB, Aly $\mathrm{AH}$. Biophotonic sensor for rapid detection of brain lesions using 1D photonic crystal. Opt Quant Electron. 2020;52:287.

7. Aly AH, Sayed FA, Elsayed HA. Defect mode tunability based on the electro-optical characteristics of the one-dimensional graphene photonic crystals. Appl Opt. 2020;59:4796-805.

8. Sayed FA, Elsayed HA, Aly AH. Optical properties of photonic crystals based on graphene nanocomposite within visible and IR wavelengths. Opt Quant Electron. 2020;52:464.

9. Surdo S, Barillaro G. On the performance of label-free biosensors based on vertical one-dimensional photonic crystal resonant cavities. Opt Express. 2015;23:9192-201.

10. Surdo S, Barillaro G. Impact of fabrication and bioassay surface roughness on the performance of label-free resonant biosensors based on one-dimensional photonic crystal microcavities. ACS Sens. 2020;5:2894-902.

11. Kang Y, Liu H. Wideband absorption in one dimensional photonic crystal with graphene-based hyperbolic metamaterials. Superlattices Microstruct. 2018;114:355-60.

12. Revathy V, Boopathi C, Selvakumar K, Wilson KSJ, Taya SA, Aly AH, et al. Nonlinear polarization in metal nanocomposite system based photonic crystals. Optik (Stuttg). 2019;176:78-84.

13. Wang Y, Chen D, Zhang G, Wang J, Tao S. A super narrow band filter based on silicon 2D photonic crystal resonator and reflectors. Opt Commun. 2016;363:13-20.

14. Seifouri M, Fallahi V, Olyaee S. Ultra-high-Q optical filter based on photonic crystal ring resonator. Photonic Netw Commun. 2018;35:225-30.

15. Liu Y, Qin F, Zhou F, Meng Q-b, Zhang D-z, Li Z-y. Ultrafast optical switching in Kerr nonlinear photonic crystals. Front Phys China. 2010;5:220-44.

16. Shirdel M, Mansouri-Birjandi MA. Photonic crystal all-optical switch based on a nonlinear cavity. Optik (Stuttg). 2016;127:3955-8.

17. Jiu-Sheng L, Han L, Le Z. Compact four-channel terahertz demultiplexer based on directional coupling photonic crystal. Opt Commun. 2015;350:248-51.

18. Rakhshani MR, Mansouri-Birjandi MA. Design and simulation of wavelength demultiplexer based on heterostructure photonic crystals ring resonators. Physica E. 2013;50:97-101.
19. Talebzadeh R, Soroosh M, Kavian YS, Mehdizadeh F. Eightchannel all-optical demultiplexer based on photonic crystal resonant cavities. Optik (Stuttg). 2017;140:331-7.

20. Kanayeva D, Bekniyazov I, Ashikbayeva Z. Detection of tuberculosis using biosensors: recent progress and future trends. Sensors \& Transducers. 2013;149:166.

21. WHO: World Health Organization. Global tuberculosis report. Geneva: WHO; 2019.

22. Srivastava SK, Van Rijn CJ, Jongsma MA. Biosensor-based detection of tuberculosis. RSC Advances. 2016;6:17759-71.

23. Pitruzzello G, Krauss TF. Photonic crystal resonances for sensing and imaging. J Opt. 2018;20:073004.

24. Zaky ZA,Aly AH. Theoretical study of a tunable low-temperature photonic crystal sensor using dielectric-superconductor nanocomposite layers. J Supercond Nov Magn. 2020;33:2983-90.

25. Abd El-Ghany SE, Noum WM, Matar Z, Zaky ZA, Aly AH. Optimized bio-photonic sensor using 1D-photonic crystals as a blood hemoglobin sensor. Phys Scr. 2020;96:035501.

26. Zaky ZA, Aly AH. Modeling of a biosensor using Tamm resonance excited by graphene. Appl Opt. 2021;60:1411-9.

27. Barillaro G, Bruschi P, Diligenti A, Nannini A. Fabrication of regular silicon microstructures by photo-electrochemical etching of silicon. Physica Status Solidi (c), 2005;2(9):3198-3202.

28. Barillaro G, Merlo S, Surdo S, Strambini LM, Carpignano F. Integrated optofluidic microsystem based on vertical highorder one-dimensional silicon photonic crystals. Microfluid Nanofluidics. 2012;12:545-52.

29. Barillaro G, Merlo S, Strambini LM. Bandgap tuning of silicon micromachined 1-D photonic crystals by thermal oxidation. IEEE J Sel Top Quantum Electron. 2008;14:1074-81.

30. Surdo S, Carpignano F, Strambini LM, Merlo S, Barillaro G. Capillarity-driven (self-powered) one-dimensional photonic crystals for refractometry and (bio) sensing applications. RSC Advances. 2014;4:51935-41.

31. Surdo S, Carpignano F, Merlo S, Barillaro G. Near-infrared silicon photonic crystals with high-order photonic bandgaps for high-sensitivity chemical analysis of water-ethanol mixtures. ACS Sens. 2018;3:2223-31.

32. Wei L, Shi J, Cheng G, Lu L, Xu H, Li Y. Pt/TiN-TiO2 catalyst preparation and its performance in oxygen reduction reaction. J Power Sources. 2020;454:227934.

33. Reddy NM, Kothandan D, Lingam SC, Ahmad A. A study on refractive index of plasma of blood of patients suffering from tuberculosis. Int. J. Technol. Eng. 2012;8:23-5.

34. Iwasaka M,Asada H. Floating photonic crystals utilizing magnetically aligned biogenic guanine platelets. Sci Rep. 2018;8:16940.

35. Hao J-J, Xie X, Gu K-D, Liu Y-J, Xia L, Yang H-W. Study on modulation of near infrared radiation based on plasma photonic crystal. Curr Appl Phys. 2020;20:961-6.

36. Biswal A, Kumar R, Nayak C, Samiappan D. n-GaAs based extrinsic Dodecanacci photonic quasicrystal. Physica B. 2020;595:412340.

37. Qutb SR, Aly AH, Sabra W. Salinity and temperature detection for seawater based on a 1D-defective photonic crystal material. Int J Mod Phys B. 2021;35(1):2150012.

38. Sharma P, Sharan P. Design of photonic crystal based ring resonator for detection of different blood constituents. Opt Commun. 2015;348:19-23.

39. Liu Y, Wang S, Biswas P, Palit P, Zhou W, Sun Y. Optofluidic vapor sensing with free-space coupled 2D photonic crystal slabs. Sci Rep. 2019;9:4209.

40. Aly AH, Zaky ZA, Shalaby AS, Ahmed AM, Vigneswaran D. Theoretical study of hybrid multifunctional one-dimensional photonic crystal as a flexible blood sugar sensor. Phys Scr. 2020;95:035510.

41. Zaky ZA, Ahmed AM, Shalaby AS, Aly AH. Refractive index gas sensor based on the Tamm state in a one-dimensional photonic crystal: theoretical optimisation. Sci Rep. 2020;10:9736.

42. Aly AH, Zaky ZA. Ultra-sensitive photonic crystal cancer cells sensor with a high-quality factor. Cryogenics. 2019;104:102991. 
43. Hu J, Sun X, Agarwal A, Kimerling LC. Design guidelines for optical resonator biochemical sensors. J Opt Soc Am B. 2009;26:1032-41.

44. White IM, Fan X. On the performance quantification of resonant refractive index sensors. Opt Express. 2008;16:1020-8.

45. El Beheiry M, Liu V, Fan S, Levi O. Sensitivity enhancement in photonic crystal slab biosensors. Opt Express. 2010;18:22702-14.

46. Ramanujam N, Patel SK, Reddy NM, Taya SA, Vigneswaran D, Rajan MM. One-dimensional ring mirror-defect photonic crystal for detection of Mycobacterium tuberculosis bacteria. Optik (Stuttg). 2019;219:165097.
47. Patel SK, Parmar J, Kosta YP, Ladumor M, Zakaria R, Nguyen TK, et al. Design of graphene metasurface based sensitive infrared biosensor. Sens Actuators A Phys. 2020;301:111767.

48. Abadla MM, Elsayed HA. Detection and sensing of hemoglobin using one-dimensional binary photonic crystals comprising a defect layer. Appl Opt. 2020;59:418-24.

49. Ramanujam NR, El-Khozondar HJ, Dhasarathan V, Taya SA, Aly AH. Design of one dimensional defect based photonic crystal by composited superconducting material for bio sensing applications. Physica B. 2019;572:42-55. 